\title{
Resonant photoproduction of ultrarelativistic electron-positron pairs on a nucleus in moderate and strong monochromatic light fields
}

\author{
Sergei P. Roshchupkin $\odot,{ }^{*}$ Nikita R. Larin $\odot,^{\dagger}$ and Victor V. Dubov ${ }^{\ddagger}$ \\ Peter the Great St.Petersburg Polytechnic University, Saint-Petersburg 195251, Russia
}

(Received 15 August 2021; accepted 24 November 2021; published 17 December 2021)

\begin{abstract}
For a complete development of quantum electrodynamics in the presence of a strong external field, proper understanding of the resonant processes and all their peculiarities is essential. We present our attempt to analytically investigate the resonant case of laser-assisted ultrarelativistic electron-positron pair photoproduction on a nucleus. The initial gamma quantum with energy $\hbar \omega_{i} \lesssim 100 \mathrm{GeV}$ and external field with intensity up to $I \sim 10^{24} \mathrm{~W} / \mathrm{cm}^{2}$ are considered. Due to the presence of the external field, the intermediate virtual particle may become real, herewith the second order process in the fine structure constant effectively reduces into the two successive first order processes. All inherent kinematics features were discussed in details and the resonant differential cross sections with simultaneous registration of the produced particle (positron for channel A or electron for channel B) energy and its outgoing angle were obtained. We established that the resonant energies of produced particles acquire two different values with respect to the positron (channel A) or electron (channel B) outgoing angle. Additionally, the certain minimal amount of absorbed wave photons is required for resonance to happen. Wherein, the resonant differential cross section significantly exceeds the corresponding one without the external field within the particular kinematic regions. Consequently, the considered process can be used as a marker for probing theoretical predictions of quantum electrodynamics with strong background electromagnetic field.
\end{abstract}

DOI: 10.1103/PhysRevD.104.116011

\section{INTRODUCTION}

Nowadays, the nonlinear phenomena of quantum electrodynamics (QED) within the strong electromagnetic field attract enormous interest [1-8] due to the development of the contemporary high-intensity laser radiation facilities [9-16] and high-energy particles sources [17-19]. The resonant behavior of the second order processes in the fine structure constant is among of such phenomena [20-26]. The feature of these processes is that the intermediate virtual particle becomes a real one, and by virtue of it the initial process of the second order effectively splits into the two successive first order processes. Wherein, the resonant differential cross section may significantly surpass the corresponding nonresonant one within the certain kinematic region. Therefore, it makes resonant processes potential candidates to become markers for probing the predictions of QED in the presence of a strong external field.

\footnotetext{
*serg9rsp@gmail.com

†larin_nr@spbstu.ru

"dubov@spbstu.ru
}

Published by the American Physical Society under the terms of the Creative Commons Attribution 4.0 International license. Further distribution of this work must maintain attribution to the author(s) and the published article's title, journal citation, and DOI. Funded by SCOAP ${ }^{3}$.
The conversion of electromagnetic radiation into the matter is one of the most intriguing phenomena since the dawn of quantum field theory. There are diverse scenarios of the electron-positron pair productions in nature [27], amid them the famous Bethe-Haitler (BH) [28] and BreitWheeler (BW) [29] processes. In turn, the former may be modified by the presence of strong external field, and one refers to it as the laser-assisted $\mathrm{BH}$ process. In the present paper, we are concerned with the resonant case of this process. Notwithstanding the large amount of fruitful researches devoted to the laser-assisted $\mathrm{BH}$ process [30-35], the complete description of this problem, especially the resonant situation, is hitherto far away from the completeness. We want explicitly to highlight previous works, where attention was paid to the resonant laserassisted $\mathrm{BH}$ process for the case of weak monochromatic $[36,37]$ and pulsed $[38,39]$ plane wave field. Within the current work, our analytical investigation is extended to the case of moderate and strong external field.

The inherent feature of the laser-assisted $\mathrm{BH}$ process is that there are two parameters that govern its behavior. The first is a classical relativistic invariant parameter, which defines the interactions of fermions with background plane wave field [7]:

$$
\eta=\frac{e F \chi}{m c^{2}}
$$


Numerically, it equals to the ratio of the field work at a wavelength to the fermion rest energy ( $e$ and $m$ are the charge and mass of fermion, $F$ and $\chi=c / \omega$ are the field strength and wavelength, $\omega$ is a wave frequency). The second is a quantum multiphoton parameter, that appears when particles interact with the Coulomb center in the presence of a plane electromagnetic wave [40]:

$$
\gamma=\eta \frac{m v c}{\hbar \omega} .
$$

Herein $v$ is the particle velocity, $c$ is the speed of light. However, this parameter (2) plays an essential role only for the case, when particles are scattered on a large angle by the Coulomb potential. Otherwise, when the scattering angle is small, this parameter does not appear [41]. Thus, the classical relativistic parameter (1) is the main parameter that determines the multiphoton processes in such situation, and moderate $(\eta \sim 1)$ and strong $(\eta \gg 1)$ fields are classified by it. Henceforth, we will employ the natural system of units $\hbar=c=1$.

\section{THE AMPLITUDE OF THE PROCESS}

We carry out all calculations within the Furry formalism [42] and take into account the interaction of produced pair with external background by exploiting the Volkov solutions [43]. In order to deduce analytical expressions for the resonant differential cross section, we adhere to the model of plane monochromatic electromagnetic wave with circular polarization, which propagates along the $z$ axes. Let us choose the corresponding four-potential in the following form:

$$
\begin{aligned}
A(\phi) & =\frac{F}{\omega}\left(e_{x} \cos \phi+\delta e_{y} \sin \phi\right), \\
\phi & =k x=\omega(t-z),
\end{aligned}
$$

where $k=(\omega, \mathbf{k})$ is the wave vector, $e_{x, y}=\left(0, \mathbf{e}_{x, y}\right)$ are the polarization four-vectors of the wave, particularly $e_{x, y}^{2}=-1,\left(e_{x, y} k\right)=k^{2}=0$ and $\delta= \pm 1$. We treat interaction with the Coulomb potential of the nucleus within the first Born approximation. Therefore, we restrict ourselves with the condition $Z \alpha / v \ll 1$ ( $Z$ is the nuclear charge, $\alpha$ is the fine structure constant).

The considered process is of the second order in the fine structure constant, consequently it is described by two Feynman diagrams (see Fig. 1), which differ from each other by the intermediate state.

The amplitude of such process can be represented as the sum over total number of absorbed (emitted) photons of external wave [25]:

$$
S=\sum_{l=-\infty}^{\infty} S_{l}
$$

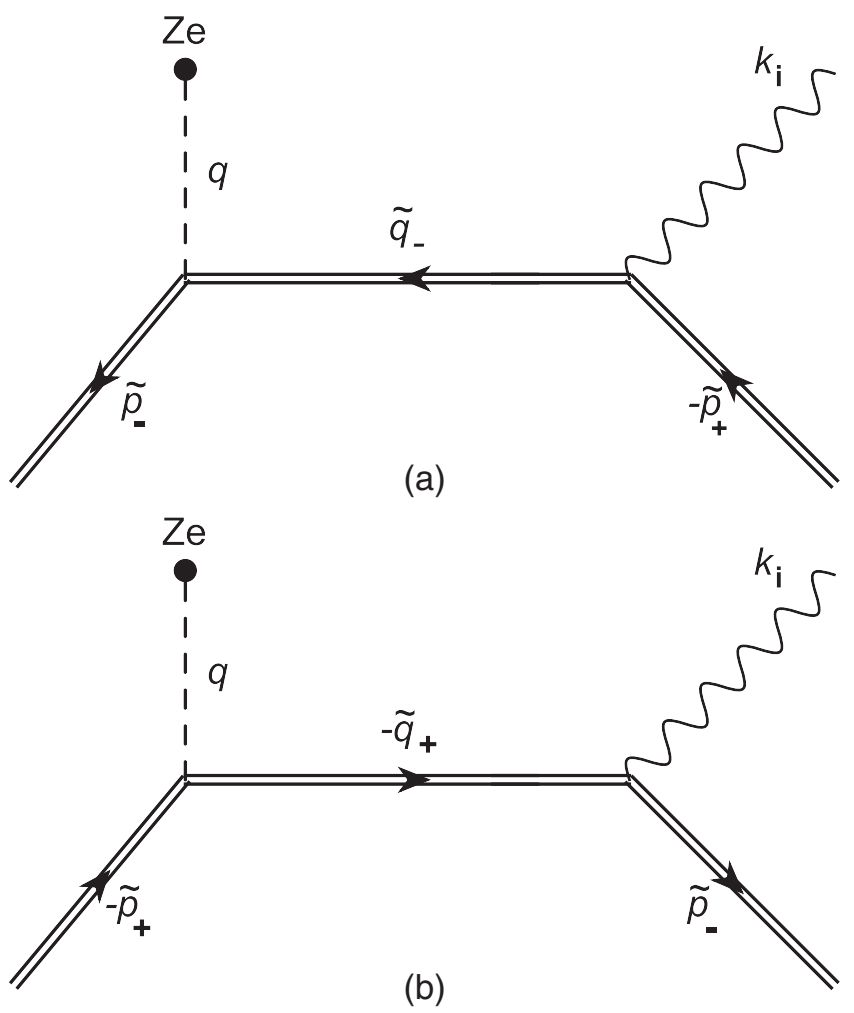

FIG. 1. Feynman diagrams of the laser-assisted BH process. Double incoming and outgoing lines correspond to the dressed electron and positron in the initial and final states. The inner lines stand for the intermediate dressed state, initial gamma quantum and pseudo photon of recoil are depicted by the wavy and dashed lines, respectively.

$$
\begin{gathered}
S_{l}=\frac{8 Z e^{3} \pi^{5 / 2}}{\sqrt{2 \tilde{E}_{+} \tilde{E}_{-} \omega_{i}}} \exp (i \psi)\left[\bar{u}_{p_{-}, \lambda} B_{l} v_{p_{+}, \lambda^{\prime}}\right] \frac{\delta\left(q^{0}\right)}{\mathbf{q}^{2}}, \\
B_{l}=\sum_{r=-\infty}^{+\infty}\left[M_{r-l}\left(\tilde{p}_{-}, \tilde{q}_{-}\right) \frac{\hat{\tilde{q}}_{-}-\frac{\eta^{2} m^{2}}{2\left(k q_{-}\right)} \hat{k}+m}{\tilde{q}_{-}^{2}-m_{*}^{2}} F_{-r}\left(\tilde{q}_{-}, \tilde{p}_{+}\right)\right. \\
\left.+M_{r-l}\left(\tilde{p}_{+}, \tilde{q}_{+}\right) \frac{\hat{\tilde{q}}_{+}-\frac{\eta^{2} m^{2}}{2\left(k q_{+}\right)} \hat{k}+m}{\tilde{q}_{+}^{2}-m_{*}^{2}} F_{-r}\left(\tilde{q}_{+}, \tilde{p}_{-}\right)\right]
\end{gathered}
$$

Hereinafter, all notations with hat imply the contraction of the corresponding vector with Dirac gamma matrices $\tilde{\gamma}^{\mu}=\left(\tilde{\gamma}^{0}, \tilde{\gamma}\right), \mu=0,1,2,3$ (e.g., $\left.\hat{k}=k_{\mu} \tilde{\gamma}^{\mu}=k_{0} \tilde{\gamma}^{0}-\mathbf{k} \tilde{\gamma}\right)$. In the expression (5) $\bar{u}_{p_{-}, \lambda}$ and $v_{p_{+}, \lambda^{\prime}}$ are free Dirac bispinors for electron in the final and positron in the initial state, respectively, and $\psi$ is an insignificant phase that does not depend on the summation index. Here we introduced the notations for the electron and positron four-quasimomenta $\tilde{p}_{ \pm}=\left(\tilde{E}_{ \pm}, \tilde{\mathbf{p}}_{ \pm}\right)$, as well as for four-quasimomenta of intermediate states $\tilde{q}_{ \pm}=\left(\tilde{E}_{ \pm}, \tilde{\mathbf{q}}_{ \pm}\right)$:

$$
\tilde{q}_{-}=-\tilde{p}_{+}+k_{i}+r k, \quad \tilde{q}_{+}=-\tilde{p}_{-}+k_{i}+r k,
$$




$$
\begin{gathered}
q=\tilde{p}_{+}+\tilde{p}_{-}-k_{i}-l k, \\
\tilde{p}_{ \pm}=p_{ \pm}+\eta^{2} \frac{m^{2}}{2\left(k p_{ \pm}\right)} k, \\
\tilde{q}_{ \pm}=q_{ \pm}+\eta^{2} \frac{m^{2}}{2\left(k q_{ \pm}\right)} k, \\
\tilde{p}_{ \pm}^{2}=m_{*}^{2}, \quad m_{*}=m \sqrt{1+\eta^{2}} .
\end{gathered}
$$

Herein $k_{i}=\omega_{i}\left(1, \mathbf{n}_{i}\right)$ is the four-momentum of the initial gamma quantum and $m_{*}$ is an effective mass of fermion within the external electromagnetic field (3). The amplitudes $M_{r-l}$ and $F_{-r}$ in the relation (6) have the following expressions:

$$
\begin{aligned}
M_{l-r}\left(\tilde{p}_{2}, \tilde{p}_{1}\right)= & a^{0} L_{r-l}\left(\tilde{p}_{2}, \tilde{p}_{1}\right)+b_{-}^{0} L_{r-l-1} \\
& +b_{+}^{0} L_{r-l+1}, \\
F_{-r}\left(\tilde{p}_{2}, \tilde{p}_{1}\right)= & (a \varepsilon) L_{-r}\left(\tilde{p}_{2}, \tilde{p}_{1}\right)+\left(b_{-} \varepsilon\right) L_{-r-1} \\
& +\left(b_{+} \varepsilon\right) L_{-r+1},
\end{aligned}
$$

where we denoted by parentheses (12) the dot product of the initial gamma quantum polarization four-vector $\varepsilon^{\mu}$ with matrices $a^{\mu}, b_{ \pm}^{\mu}$, that are defined in the following way:

$$
\begin{gathered}
a^{\mu}=\tilde{\gamma}^{\mu}+\eta^{2} \frac{m^{2}}{2\left(k \tilde{p}_{1}\right)\left(k \tilde{p}_{2}\right)} k^{\mu} \hat{k}, \\
b_{ \pm}^{\mu}=\frac{1}{4} \eta m\left[\frac{\hat{\varepsilon}_{ \pm} \hat{k} \gamma^{\mu}}{\left(k \tilde{p}_{2}\right)}+\frac{\gamma^{\mu} \hat{k} \hat{\varepsilon}_{ \pm}}{\left(k \tilde{p}_{1}\right)}\right], \quad \hat{\varepsilon}_{ \pm}=\hat{e}_{x} \pm i \delta \hat{e}_{y},
\end{gathered}
$$

The special functions $L_{r-l}\left(\tilde{p}_{2}, \tilde{p}_{1}\right), L_{-r}\left(\tilde{p}_{2}, \tilde{p}_{1}\right)$ and their arguments are given by the expressions [44]:

$$
\begin{gathered}
L_{n}\left(\tilde{p}_{2}, \tilde{p}_{1}\right)=\exp \left(-i n \chi_{\tilde{p}_{2} \tilde{p}_{1}}\right) J_{n}\left(\gamma_{\tilde{p}_{2} \tilde{p}_{1}}\right), \\
\tan \chi_{\tilde{p}_{2} \tilde{p}_{1}}=\delta \frac{\left(e_{y} Q_{\tilde{p}_{2} \tilde{p}_{1}}\right)}{\left(e_{x} Q_{\tilde{p}_{2} \tilde{p}_{1}}\right)}, \quad Q_{\tilde{p}_{2} \tilde{p}_{1}}=\frac{\tilde{p}_{2}}{\left(k \tilde{p}_{2}\right)}+\frac{\tilde{p}_{1}}{\left(k \tilde{p}_{1}\right)}, \\
\gamma_{\tilde{p}_{2} \tilde{p}_{1}}=\eta m \sqrt{-Q_{\tilde{p}_{2} \tilde{p}_{1}}^{2}} \cdot
\end{gathered}
$$

We note that $\left(k \tilde{p}_{1,2}\right)=\left(k p_{1,2}\right)$ and thus, to obtain the appropriate expressions for the channel A (i.e., for the first term in (6)) we need to replace $\tilde{p}_{1} \rightarrow-\tilde{p}_{+}, \tilde{p}_{2} \rightarrow \tilde{q}_{-}$for $F_{-r}\left(\tilde{p}_{2}, \tilde{p}_{1}\right)$ and $\tilde{p}_{1} \rightarrow \tilde{q}_{-}, \tilde{p}_{2} \rightarrow \tilde{p}_{-}$for $M_{l-r}\left(\tilde{p}_{2}, \tilde{p}_{1}\right)$ in the relations (13)-(17). For the channel B (i.e., for the second term in (6)) one has to act in similar way and make the replacement $\tilde{p}_{1} \rightarrow \tilde{p}_{-}, \tilde{p}_{2} \rightarrow-\tilde{q}_{+}$for $F_{-r}\left(\tilde{p}_{2}, \tilde{p}_{1}\right)$ and $\tilde{p}_{1} \rightarrow-\tilde{q}_{+}, \tilde{p}_{2} \rightarrow-\tilde{p}_{+}$for $M_{l-r}\left(\tilde{p}_{2}, \tilde{p}_{1}\right)$. It is important to emphasize, that obtained amplitude (5)-(17) is valid for the arbitrary intensities and frequencies of the plane monochromatic wave with circular polarization (3).

\section{POLES OF THE AMPLITUDE IN A STRONG FIELD}

In presence of the external electromagnetic field (3) the intermediate particle momentum may satisfy its dispersion relation:

$$
\begin{aligned}
& \tilde{q}_{-}^{2}=m_{*}^{2}, \\
& \tilde{q}_{+}^{2}=m_{*}^{2} .
\end{aligned}
$$

Such behavior is caused by the quasi-discrete energy spectrum of fermion propagating within the plane electromagnetic wave [45]. Due to that fact, one may interpret it as the reduction of the second order process (see Fig. 1) into the two successive first order processes in fine structure constant (see Fig. 2). There is four-quasimomentum conservation law, which can be written for both channels in every vertex (see Fig. 2) in the following way:

$$
\begin{gathered}
k_{i}+r k=\tilde{p}_{+}+\tilde{q}_{-}, \\
q=\tilde{q}_{-}-\tilde{p}_{-}+(l-r) k
\end{gathered}
$$
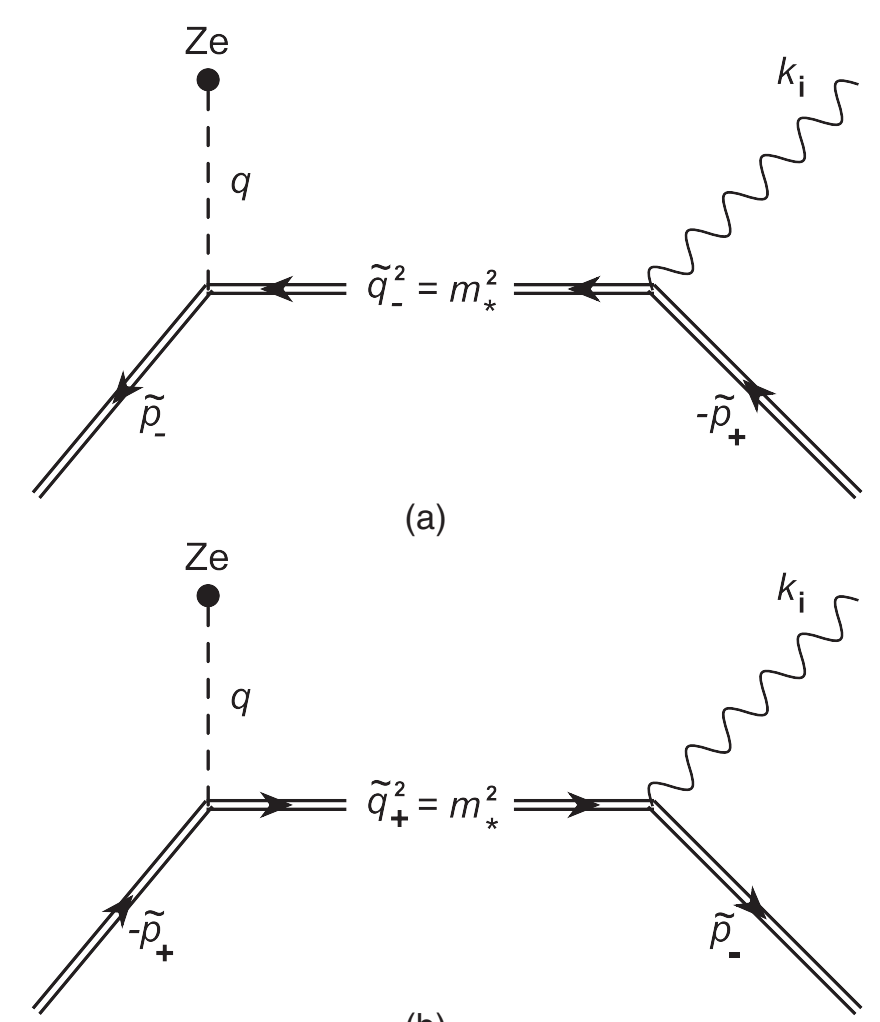

(b)

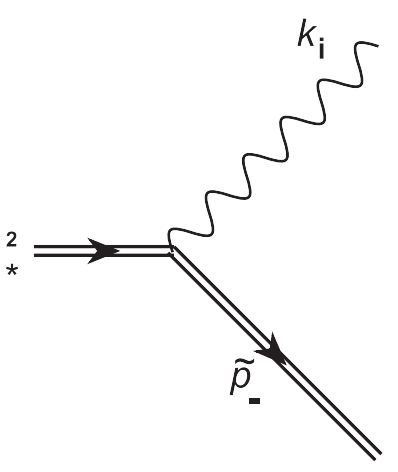

FIG. 2. Feynman diagrams of the resonant laser-assisted BH process. 
and

$$
\begin{gathered}
k_{i}+r k=\tilde{p}_{-}+\tilde{q}_{+}, \\
q=\tilde{q}_{+}-\tilde{p}_{+}+(l-r) k .
\end{gathered}
$$

Insofar as $\tilde{p}_{ \pm}^{2}=\tilde{q}_{ \pm}^{2}=m_{*}^{2}$, and $k^{2}=k_{i}^{2}=0$, the equalities (20) and (22) are fulfilled only for the $r \geq 1$. This fact in conjunction with the form of amplitude (5), (6) (see also Fig. 2) allow us to conclude that $F_{-r}$ (12) represents the amplitude of the laser-stimulated BW process [7] with absorption of $r$ wave photons. In turn, $M_{r-l}$ (11) is nothing but the amplitude of laser-assisted Mott scattering of electron (channel A) or positron (channel B) on a nucleus with the absorption (emission) of $|r-l|$ wave photons [6]. Hence, that verifies that in the absence of interference, the initial second order process in the fine structure constant effectively reduces into the two successive first order processes, as was mentioned above.

The thorough examination of the resonant conditions (18), (19) and conservation laws (20)-(23) shows us that for resonance to occur, one of the possibilities is to require the ultrarelativistic energies of produced particles and thus, the sufficient energy of the initial gamma quantum. Moreover, the resonant kinematics region is confined with the configuration, where all produced particles propagate within the narrow cone with initial gamma quantum direction. Additionally, we demand the directions of initial gamma quantum and external wave propagation do not coincidence, otherwise resonances are merely impossible:

$$
\begin{gathered}
\omega_{i} \gg m, \\
\theta_{i \pm}=\left(\mathbf{k}_{i}, \mathbf{p}_{ \pm}\right) \ll 1, \quad \bar{\theta}_{ \pm}=\left(\mathbf{p}_{-}, \mathbf{p}_{+}\right) \ll 1, \\
\theta_{i}=\left(\mathbf{k}_{i}, \mathbf{k}\right) \sim 1, \quad \theta_{ \pm}=\left(\mathbf{k}, \mathbf{p}_{ \pm}\right) \sim 1 .
\end{gathered}
$$

In a matter of fact, the condition (24) has to be rewritten for the case of strong field, when the classical parameter becomes not small $\eta \gtrsim 1$. We face the necessity to replace the particle mass with the effective mass [46]. Hence, the condition (24) takes form:

$$
\frac{\omega_{i}}{m_{*}}=\frac{\omega_{i}}{m \sqrt{1+\eta^{2}}} \sim \begin{cases}\omega_{i} / m \gg 1, & \text { if } \eta \ll 1 \\ \omega_{i} /(\eta m) \gg 1, & \text { if } \eta \gtrsim 1\end{cases}
$$

From the second string in the condition (26) we obtain the restriction on the maximum intensity of the external field:

$$
\eta \ll \eta_{\max }=\frac{\omega_{i}}{m} \gg 1 .
$$

By similar reasoning, we formulate the new ultrarelativistic condition for the produced particles:

$$
\frac{\tilde{E}_{ \pm}}{m_{*}} \approx \frac{E_{ \pm}}{m \sqrt{1+\eta^{2}}} \sim \begin{cases}E_{ \pm} / m \gg 1, & \text { if } \eta \ll 1 \\ E_{ \pm} /(\eta m) \gg 1, & \text { if } \eta \gtrsim 1\end{cases}
$$

Deliberately, throughout our research, we consider the initial gamma quantum energy $\omega_{i} \lesssim 100 \mathrm{GeV}$. This value leads us to the estimation of the classical invariant parameter $\eta \ll \eta_{\max } \sim 10^{5}$, that corresponds to $F \ll F_{\max } \sim$ $10^{15} \mathrm{~V} / \mathrm{cm}\left(I \ll I_{\max } \sim 10^{28} \mathrm{~W} / \mathrm{cm}^{2}\right)$ for the optical frequency range. Therefore, all further results are valid for sufficiently large intensity. However, they are still not applicable to the fields of the critical Schwinger limit $F_{\text {cr }} \approx 1.3 \times 10^{16} \mathrm{~V} / \mathrm{cm}[47]$.

With use of relations (18) and (20) we can derive the expression for the resonant positron energy in channel A:

$$
x_{\eta+(r)}=\frac{\kappa_{\eta(r)} \pm \sqrt{\kappa_{\eta(r)}\left(\kappa_{\eta(r)}-1\right)-\delta_{\eta+}^{2}}}{2\left(\kappa_{\eta(r)}+\delta_{\eta+}^{2}\right)},
$$

Analogously, relations (19) and (22) help us to deduce the expression for resonant electron energy for channel B:

$$
x_{\eta-(r)}=\frac{\kappa_{\eta(r)} \pm \sqrt{\kappa_{\eta(r)}\left(\kappa_{\eta(r)}-1\right)-\delta_{\eta-}^{2}}}{2\left(\kappa_{\eta(r)}+\delta_{\eta-}^{2}\right)} .
$$

Here we introduced notations:

$$
\begin{gathered}
x_{\eta \pm(r)}=\frac{E_{\eta \pm(r)}}{\omega_{i}}, \quad \kappa_{\eta(r)}=\frac{r}{r_{\eta}}, \quad \delta_{\eta \pm}=\frac{\omega_{i} \theta_{i \pm}}{2 m_{*}} \\
r_{\eta}=\frac{m_{*}^{2}}{\omega_{i} \omega \sin ^{2}\left(\theta_{i} / 2\right)} .
\end{gathered}
$$

From relations (29) and (30) we can conclude, that resonant energies of positron (channel A) and electron (channel B) are determined by the corresponding outgoing angle and quantum parameter $\kappa_{\eta}(r)$. In turn, the latter represents the ratio of absorbed wave photons $r$ to the minimum required amount of them $r_{\eta}$, which is defined by the experimental setup [see (32)]. It follows from the (29) and (30), that $\kappa_{\eta(r)}$ has to be greater than one, and hence:

$$
r \geq r_{\min } ; \quad r_{\min }=\left\lceil r_{\eta}\right\rceil .
$$

Henceforth, we will use for assessments the certain set of parameters: $\omega_{i}=50 \mathrm{GeV}, \omega=1 \mathrm{eV}, \theta_{i}=\pi$. For such set, it follows:

$$
r_{\eta} \approx 5.2\left(1+\eta^{2}\right) .
$$

One can see that in strong fields $(\eta \gg 1)$, the resonant process involves large number of absorbed wave photons $\left(r_{\eta} \approx \eta^{2} \gg 1\right)$. By the definition of $r_{\eta}$ it follows, as well, that the number of minimum required absorbed photons 


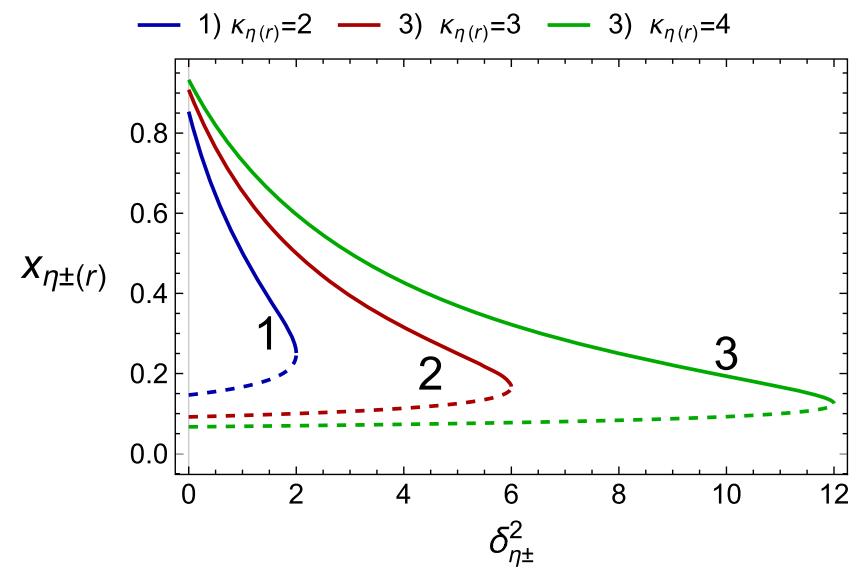

FIG. 3. The resonant positron (channel A) and electron (channel B) energies as functions of the corresponding outgoing angle, plotted for the different values of $\kappa_{\eta(r)}$. Solid lines represent the high-energy solution, meantime dashed lines stand for the lowenergy solutions (29), (30).

increases proportional to intensity $\left(r_{\eta} \sim \eta^{2} \sim I\left(\mathrm{~W} \mathrm{~cm}^{-2}\right)\right)$. We underline that all obtained expressions (29)-(32) are in complete agreement with the weak field limit $\eta \ll 1$, particularly the parameter $r_{\eta}$ reduces to the threshold energy for initial gamma quantum [36].

Another peculiarity of the resonant behavior is that energies of produced particles may take two different values for the particular outgoing angle (see Fig. 3). From now on, we will refer to the expressions with $\langle+\rangle\rangle$ sign in numerators of (29) and (30) as high-energy solutions and to the expressions with $\langle\langle-\rangle\rangle$ sign as lowenergy solutions. Also, the outgoing angle of particle (positron for channel A and electron for channel B) is enclosed in the interval, which is defined by the following inequality:

$$
0 \leq \delta_{\eta \pm}^{2} \leq \delta_{\eta \pm \max }^{2}=\kappa_{\eta(r)}\left(\kappa_{\eta(r)}-1\right) .
$$

Inasmuch there are no intersections between energy's value with different $\kappa_{\eta}(r)$ within the frame of particular channel (see Fig. 3), we can distinguish one process with different number of absorbed photons from another and thus, they do not interfere.

\section{THE RESONANT DIFFERENTIAL CROSS SECTION IN THE ULTRARELATIVISTIC ENERGY LIMIT}

As long as we confine ourselves with the condition (27), we may neglect the second and the third term in fermion scattering amplitude (11) $\quad\left(\left|b_{ \pm}^{0}\right| \lesssim \eta m / \omega_{i} \ll 1\right.$, see Eqs. (14) and (26)). As a result, the expression for $M_{r-l}$ essentially simplifies:

$$
M_{r-l}=\exp \left[-i(r-l) \chi_{\tilde{p}_{-} \tilde{q}_{-}}\right] J_{r-l}\left[\gamma\left(\tilde{p}_{-}, \tilde{q}_{-}\right)\right] \gamma^{0} .
$$

For the conciseness, we represent our derivations for the channel A. To obtain the corresponding relations for channel $\mathrm{B}$, one must substitute $\tilde{q}_{-} \rightarrow \tilde{q}_{+}, \tilde{p}_{-} \rightarrow-\tilde{p}_{+}$. In addition, we introduce the subscript $\langle\langle+\rangle\rangle$ for further expressions to signify, that within the channel A all deduced quantities are defined by the positron outgoing angle, in contrast to the channel B, where we use the subscript $\langle\langle-\rangle\rangle$ to accentuate the similar role of the electron outgoing angle. The influence of interference between different channels is left out of our consideration. Nevertheless, we emphasize that there is indeed interference in resonance case, and its impact requires further investigation.

We perform the standard procedure [48] to derive resonant differential cross section for the unpolarized particles from the amplitude (4)-(6), (11), (12), (36):

$$
d \sigma_{+(l, r)}=\frac{2}{\pi^{2}} d \mathrm{M}_{+(l-r)} \frac{m^{2} E_{-}}{\left|\tilde{q}_{-}^{2}-m_{*}^{2}\right|^{2}} d \mathrm{P}_{+(r)} .
$$

Herein, $d \mathrm{M}_{+(l-r)}$ represents differential cross section of the intermediate electron scattering on the nucleus with emission (absorption) of $|l-r|$ wave photons (laser-assisted Mott scattering) [49]:

$$
\begin{aligned}
d \mathrm{M}_{+(l-r)}= & Z^{2} r_{e}^{2} \frac{m^{2}}{\mathbf{q}^{4}} J_{l-r}^{2}\left(\gamma_{\tilde{p}_{-}, \tilde{q}_{-}}\right) \\
& \times \delta\left[\tilde{q}_{-}^{0}-\tilde{E}_{-}+(l-r) \omega\right] d^{3} \tilde{p}_{-},
\end{aligned}
$$

where transferred to nucleus momentum $\mathbf{q}$ and argument of the Bessel function have the following forms:

$$
\begin{aligned}
\mathbf{q} & =\tilde{\mathbf{q}}_{-}-\tilde{\mathbf{p}}_{-}+(l-r) \mathbf{k}, \\
\gamma_{\tilde{p}_{-}, \tilde{q}_{-}} & =\eta m \sqrt{-Q_{\tilde{p}_{-}, \tilde{q}_{-}}^{2}} \\
Q_{\tilde{p}_{-}, \tilde{q}_{-}} & =\frac{\tilde{p}_{-}}{\left(k \tilde{p}_{-}\right)}-\frac{\tilde{q}_{-}}{\left(k \tilde{q}_{-}\right)} .
\end{aligned}
$$

Function $d \mathrm{P}_{+(r)}$ determines the differential probability (per unit of time) of the laser-stimulated BW process with the absorption of $r$ wave photons [7]:

$$
d \mathrm{P}_{+(r)}=\frac{\alpha}{\omega_{i} E_{+}} P\left(u_{\eta+(r)}, v_{\eta(r)}\right) d^{3} \tilde{p}_{+},
$$

here

$$
\begin{gathered}
P\left(u_{\eta+(r)}, v_{\eta(r)}\right) \\
=J_{r}^{2}\left(\gamma_{\tilde{q}_{-} \tilde{p}_{+}}\right)+\eta^{2}\left(2 u_{\eta+(r)}-1\right)\left[\left(\frac{r^{2}}{\gamma_{\tilde{q}_{-} \tilde{p}_{+}}^{2}}-1\right) J_{r}^{2}+J_{r}^{\prime 2}\right], \\
\gamma_{\eta+(r)}=2 r \frac{\eta}{\sqrt{1+\eta^{2}}} \sqrt{\frac{u_{\eta+(r)}}{v_{\eta(r)}}\left(1-\frac{u_{\eta+(r)}}{v_{\eta(r)}}\right),}
\end{gathered}
$$




$$
\begin{gathered}
u_{\eta+(r)}=\frac{\left(k k_{i}\right)^{2}}{4\left(k q_{-}\right)\left(k p_{+}\right)} \approx \frac{1}{4 x_{\eta+(r)}\left(1-x_{\eta+(r)}\right)}, \\
v_{\eta(r)}=r \frac{\left(k k_{i}\right)}{2 m_{*}^{2}}=\kappa_{\eta(r)} .
\end{gathered}
$$

With allowance for (28), it is permissible to put $d^{3} \tilde{p}_{-} \approx$ $d^{3} p_{-}=E_{-}^{2} d E_{-} d \Omega_{-}$in the cross section (38) and easily carry out the integration with respect to the electron energy. Afterwards, the resonant differential cross section (38) represents the product of differential probability of the laser-stimulated BW process and differential cross section of the laser-assisted Mott scattering.

The appearance of the resonant infinity is caused by the idealized spatial and temporal dependence of the external filed, which allows analytical investigation. Complete treatment of such kind divergences involves cumbersome calculations of radiative corrections to the fermion propagator [50,51]. We also bring to attention, that resonant divergence disappears in natural way if one considers the finite size and duration of the external laser pulse [38,52-54]. In the present paper, in turn, we achieve the elimination of the resonant infinity in channels $\mathrm{A}$ and $\mathrm{B}$ by means of the renowned Breit-Wigner procedure [21,55-57]:

$$
\begin{gathered}
m_{*} \rightarrow \mu_{*}=m_{*}-i \Gamma_{\eta+(r)}, \quad \Gamma_{\eta+(r)}=\frac{\tilde{q}_{-}^{0}}{2 m_{*}} W\left(r_{\eta}\right), \\
W\left(r_{\eta}\right)=\frac{\alpha m^{2}}{4 \pi \tilde{q}_{-}^{0}} \mathrm{~K}\left(r_{\eta}\right), \quad \mathrm{K}\left(r_{\eta}\right)=\sum_{n=0}^{\infty} \mathrm{K}_{n}\left(r_{\eta}\right), \\
\mathrm{K}_{n}\left(r_{\eta}\right)=\int_{0}^{n / r_{\eta}} \frac{d u}{(1+u)^{2}} K\left(u, \frac{n}{r_{\eta}}\right), \\
K\left(u, \frac{n}{r_{\eta}}\right)=-J_{n}^{2}(z)+\eta^{2}\left(1+\frac{u^{2}}{2(1+u)}\right) \\
\times\left[\left(\frac{n^{2}}{z^{2}}-1\right) J_{n}^{2}+J_{n}^{\prime 2}\right], \\
z=2 n \frac{\eta}{\sqrt{1+\eta^{2}}} \sqrt{u \frac{n}{r_{n}}\left(1-u \frac{n}{r_{n}}\right)},
\end{gathered}
$$

where function $\mathrm{K}\left(r_{\eta}\right)$ is the total probability (per unit of time) of the laser-stimulated Compton scattering on the intermediate particle [7]. Herein, we eliminated resonant infinity with use of the first leading radiative correction to the electron propagator, which imaginary part is closely related to the probability of the laser-stimulated emission of gamma quantum [58]. In so doing, we have to ensure that $\alpha \chi^{2 / 3} \ll 1$ (where $\chi=\eta\left(k q_{-}\right) / m^{2}$ is the so-called quantum nonlinearity parameter [59]). In fact, considering our initial parameters, this condition is fulfilled while $I \sim 10^{22} \mathrm{~W} / \mathrm{cm}^{2}$ or less.
Therefore, the obtained results for the case $I \sim 10^{24} \mathrm{~W} / \mathrm{cm}^{2}$ are of a qualitative nature. Given the relations (46) and (47), the resonant denominator for channel A can be rewritten as follows:

$\left|\tilde{q}_{-}^{2}-\mu_{*}^{2}\right|^{2}=16 m_{*}^{4} x_{\eta+(r)}^{2}\left[\left(\delta_{\eta+}^{2}-\delta_{\eta+(r)}^{2}\right)^{2}+\Upsilon_{\eta+(r)}^{2}\right]$.

Here $\Upsilon_{\eta+(r)}$ is the angular resonant width:

$$
\Upsilon_{\eta+(r)}=\frac{\alpha}{16 \pi\left(1+\eta^{2}\right)} \frac{1}{x_{\eta+(r)}} \mathrm{K}\left(r_{\eta}\right) .
$$

In the Eq. (51) parameter $\delta_{\eta+(r)}^{2}$ is related to the positron resonant energy by the formula (29), meanwhile parameter $\delta_{\eta+}^{2}$ varies independently.

Further calculations with use of the relations (26)-(30) and (51) lead us to the following expressions for the differential cross sections for channel $\mathrm{A}$ and $\mathrm{B}$ :

$$
\begin{aligned}
\frac{d \sigma_{\eta \pm(l, r)}}{d x_{\eta \pm(r)} d \delta_{\eta \pm}^{2}}= & \frac{\left(Z^{2} \alpha r_{e}^{2}\right)}{\pi\left(1+\eta^{2}\right)^{2}} \frac{J_{l-r}^{2}\left(\alpha_{\eta \pm(r)}\right)}{g_{ \pm}^{4}} \\
& \times \frac{\left(1-x_{\eta \pm(r)}\right)^{3}}{\left[\left(\delta_{\eta \pm}^{2}-\delta_{\eta \pm(r)}^{2}\right)^{2}+\Upsilon_{\eta+(r)}^{2}\right] x_{\eta \pm(r)}} \\
& \times P\left(u_{\eta \pm(r)}, \kappa_{\eta(r)}\right) d \delta_{\eta \mp}^{2} d \varphi,
\end{aligned}
$$

where

$$
g_{ \pm}^{2}=g_{\eta 0}+\left(\frac{m_{*}}{2 \omega_{i}}\right)^{2} g_{\eta \pm(r, l)}
$$

We bring to attention, that as we mentioned above, subscript "+" stands for the channel A likewise, subscript " - " respect to the channel B in (53), (54). Herein $\varphi$ is the angle between planes $\left(\mathbf{k}_{i}, \mathbf{p}_{+}\right)$and $\left(\mathbf{k}_{i}, \mathbf{p}_{-}\right)$. Similarly to the channel $\mathrm{A}$, the parameter $\delta_{\eta-(r)}^{2}$ is expressed via the resonant energy of electron for channel B (30), meantime $\delta_{\eta-}^{2}$ varies independently. The relativistic invariant parameter $u_{\eta-(r)}$ and the resonant width for channel B obey to relations:

$$
\begin{gathered}
u_{\eta-(r)}=\frac{\left(k k_{i}\right)^{2}}{4\left(k q_{+}\right)\left(k p_{-}\right)} \approx \frac{1}{4 x_{\eta-(r)}\left(1-x_{\eta-(r)}\right)}, \\
\Upsilon_{\eta-(r)}=\frac{\alpha}{16 \pi\left(1+\eta^{2}\right)} \frac{1}{x_{\eta-(r)}} \mathrm{K}\left(r_{\eta}\right) .
\end{gathered}
$$

The function $P\left(u_{\eta-(r)}, r / r_{\eta}\right)$ has the form likewise to channel A (42), except the substitution $u_{\eta+(r)} \rightarrow u_{\eta-(r)}$. The impact of transferred to nucleus momentum is contained in the functions $g_{+}^{2}$ (channel A) and $g_{-}^{2}$ (channel B) (54), where we took into account the influence of corrections proportional to $m_{*}^{2} / \omega_{i}^{2}$ : 


$$
\begin{gathered}
g_{\eta 0}=\tilde{\delta}_{\eta+}^{2}+\tilde{\delta}_{\eta-}^{2}+2 \tilde{\delta}_{\eta+} \tilde{\delta}_{\eta-} \cos \varphi \\
\tilde{\delta}_{\eta \pm}=2 x_{\eta \pm(r)} \delta_{\eta \pm} \\
g_{\eta \pm(r, l)}= \\
g_{\eta \pm(r, l)}^{(0)}+\frac{1}{1+\eta^{2}} g_{\eta \pm(r, l)}^{(1)}+\frac{1}{\left(1+\eta^{2}\right)^{2}} g_{\eta \pm(r, l)}^{(2)} \\
g_{\eta \pm(r, l)}^{(0)}=\frac{\tilde{\delta}_{\eta \pm}^{2}}{3} \frac{x_{\eta \pm(r)}^{2}\left(1-x_{\eta \pm(r)}\right)^{2}-\left(1-x_{\eta \pm(r)}\right)^{3}-x_{\eta \pm(r)}^{3}}{x_{\eta \pm(r)}^{3}\left(1-x_{\eta \pm(r)}\right)^{3}} \\
-\frac{4 \beta_{\eta \pm(l, r)} \tilde{\delta}_{\eta \pm}^{2}}{x_{\eta \pm(r)}\left(1-x_{\eta \pm(r)}\right)}, \\
g_{\eta \pm(r, l)}^{(1)}=2 \tilde{\delta}_{\eta \pm}^{2} \frac{x_{\eta \pm(r)}^{3}\left(2-x_{\eta \pm(r)}\right)-\left(1-x_{\eta \pm(r)}\right)^{4}}{x_{\eta \pm(r)}^{3}\left(1-x_{\eta \pm(r)}\right)^{3}} \\
+\frac{4 \beta_{\eta \pm(l, r)}}{x_{\eta \pm(r)}\left(1-x_{\eta \pm(r)}\right)} \\
\beta_{\eta \pm(l, r)}=\frac{l}{r_{\eta}}-\frac{1}{4} \frac{\eta^{2}}{1+\eta^{2}} \frac{1}{x_{\eta \pm(r)}\left(1-x_{\eta \pm(r)}\right)} \\
g_{\eta \pm(r, l)}^{(2)}=\frac{\left(1-x_{\eta \pm(r)}\right)^{3}+x_{\eta \pm(r)}^{3}-\left(1-2 x_{\eta \pm(r)}\right)^{2}}{x_{\eta \pm(r)}^{3}\left(1-x_{\eta \pm(r)}\right)^{3}}
\end{gathered}
$$

The arguments of the Bessel functions that define the processes of emission or absorption of $|l-r|$ wave photons during the scattering of intermediate fermion on the nucleus for channel A and B have the form:

$$
\alpha_{\eta \pm(r)} \approx 2 r_{\eta} \frac{\eta}{\sqrt{1+\eta^{2}}} \sqrt{g_{\eta 0}^{2}}
$$

As we have stated before, the corrections of the magnitude of $m_{*}^{2} / \omega_{i}^{2}$ were introduced in transmitted momentum (54). These corrections are of the great importance for the certain kinematic regions. Namely, they make the dominant contribution to the differential cross section under the conditions:

$$
|\varphi-\pi| \lesssim \frac{m_{*}}{\omega_{i}} \ll 1, \quad\left|\tilde{\delta}_{\eta+}-\tilde{\delta}_{\eta-}\right| \lesssim \frac{m_{*}}{\omega_{i}} \ll 1
$$

Under such conditions, the function $g_{\eta 0}$ tends to zero and consequently, there is a sharp maximum in the differential cross section. This notorious behavior of the differential cross section in the ultrarelativistic limit is typical for the long-range Coulomb potential [48]. We perform the saddle point method to integrate resonant differential cross sections (53) within the vicinity of maxima points (64):

$$
\begin{aligned}
& \frac{d \sigma_{\eta+(l, r)}}{d x_{\eta+(r)} d \delta_{\eta+}^{2}} \\
& \quad=\frac{\left(Z^{2} \alpha r_{e}^{2}\right)}{4 \pi\left(1+\eta^{2}\right)^{2}} \frac{\left(1-x_{\eta+(r)}\right) P\left(u_{\eta+(r)}, \kappa_{\eta(r)}\right)}{\left[\left(\delta_{\eta+}^{2}-\delta_{\eta+(r)}^{2}\right)^{2}+\Upsilon_{\eta+(r)}^{2}\right] x_{\eta+(r)}} C_{\eta+(l, r)},
\end{aligned}
$$

where

$$
\begin{aligned}
C_{\eta+(l, r)}= & \int_{0}^{2 \pi} d \varphi \int_{0}^{\infty} J_{l-r}^{2}\left(\alpha_{\eta+(r)}\right) \\
& \times\left[g_{\eta 0}^{2}+\left(\frac{m_{*}}{2 \omega_{i}}\right)^{2} g_{\eta+(r, l)}\right]^{-2} d \tilde{\delta}_{\eta-}^{2} .
\end{aligned}
$$

The integrand in (66) has an abrupt maximum within the interval (64). Herewith, the function $J_{l-r}^{2}\left(\alpha_{\eta+(r)}\right) \lesssim 1$ and we are allowed to take it out of the integral

$$
\begin{aligned}
C_{\eta+(l, r)} \approx & \left.J_{l-r}^{2}\left(\alpha_{\eta+(r)}\right)\right|_{\substack{\varphi=\pi \\
\tilde{\delta}_{\eta-\bar{\delta}} \tilde{\delta}_{\eta_{+}}}} \\
& \times \int_{0}^{2 \pi} d \varphi \int_{0}^{\infty} d \tilde{\delta}_{\eta-}^{2} \exp \left[f_{\eta}\left(\varphi, \tilde{\delta}_{\eta-}\right)\right],
\end{aligned}
$$

here

$$
\begin{aligned}
f_{\eta}\left(\varphi, \tilde{\delta}_{\eta-}\right) & =-2 \ln \left(g_{\eta 0}^{2}+d_{\eta+}\right), \\
d_{\eta+} & =\left(\frac{m_{*}}{2 \omega_{i}}\right)^{2} g_{\eta+(r, l)} .
\end{aligned}
$$

We employ the Taylor expansion of the $f_{\eta}$ in the vicinity of the point $\varphi=\pi, \tilde{\delta}_{\eta-}=\tilde{\delta}_{\eta+}$

$$
\begin{aligned}
f_{\eta}\left(\varphi, \tilde{\delta}_{\eta-}\right) \approx & -2 \ln \left(d_{\eta+}\right)-\frac{2 \tilde{\delta}_{\eta+}^{2}}{d_{\eta+}}(\varphi-\pi)^{2} \\
& -\frac{1}{2 d_{\eta+} \tilde{\delta}_{\eta+}^{2}}\left(\tilde{\delta}_{\eta-}^{2}-\tilde{\delta}_{\eta+}^{2}\right)^{2} .
\end{aligned}
$$

Eventually, we perform integration (67) with use of the expansion (69)

$$
C_{\eta+(l, r)} \approx \frac{\pi}{g_{\eta+(l, r)}}\left(\frac{2 \omega_{i}^{2}}{m_{*}^{2}}\right) J_{l-r}^{2}(0)=\frac{4 \pi}{g_{\eta+(r)}}\left(\frac{\omega_{i}^{2}}{m_{*}^{2}}\right) .
$$

In so doing, we take into account that the argument of Bessel function (63) is virtually zero and thus, the Bessel function itself is not zero only for the case $l=r$. In other words, the most probable situation is the scattering of ultrarelativistic fermion on the nucleus without absorption or emission of wave photons [36,41]. Ultimately, the differential cross section for both channels may be written in the following way: 

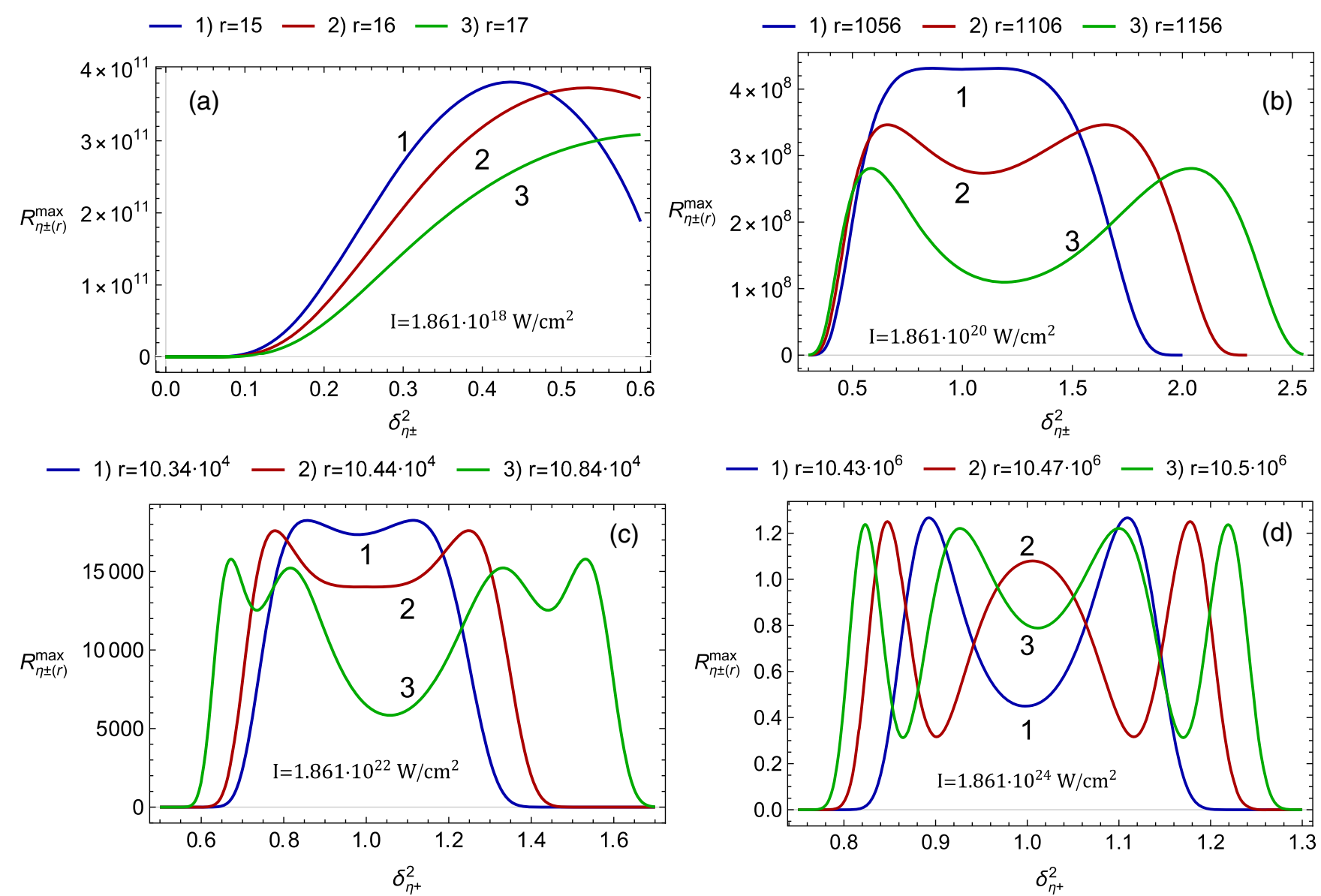

FIG. 4. The angular distribution of the maximum resonant differential cross section (73) (in $Z^{2} \alpha r_{e}^{2}$ units) for the certain parameters (34) and different intensities.

$$
\begin{aligned}
\frac{d \sigma_{\eta \pm(r)}}{d x_{\eta \pm(r)} d \delta_{\eta \pm}^{2}}= & \left(\frac{\omega_{i}}{m_{*}}\right)^{2} \frac{\left(Z^{2} \alpha r_{e}^{2}\right)}{\left(1+\eta^{2}\right)^{2}} \\
& \times \frac{\left(1-x_{\eta \pm(r)}\right)}{g_{\eta \pm(r)}\left[\left(\delta_{\eta \pm}^{2}-\delta_{\eta \pm(r)}^{2}\right)^{2}+\Upsilon_{\eta \pm(r)}^{2}\right] x_{\eta \pm(r)}} \\
& \times P\left(u_{\eta \pm(r)}, \kappa_{\eta(r)}\right)
\end{aligned}
$$

Herein the functions $g_{\eta+(r)}$ and $g_{\eta-(r)}$ are defined by the expressions (58)-(62), but instead of the index $l$ one should write $r$. When the following conditions are met

$$
\begin{aligned}
\left(\delta_{\eta+}^{2}-\delta_{\eta+(r)}^{2}\right)^{2} & \ll \Upsilon_{\eta+(r)}^{2}, \\
\left(\delta_{\eta-}^{2}-\delta_{\eta-(r)}^{2}\right)^{2} & \ll \Upsilon_{\eta-(r)}^{2}
\end{aligned}
$$

we obtain the maximum resonant differential cross section for channels $\mathrm{A}$ and $\mathrm{B}$ :

$$
R_{\eta \pm(r)}^{\max }=\frac{d \sigma_{\eta \pm(r)}^{\max }}{d x_{\eta \pm(r)} d \delta_{\eta \pm}^{2}}=\left(Z^{2} \alpha r_{e}^{2}\right) D_{\eta i} F_{\eta \pm(r)}
$$

The functions $F_{\eta+(r)}$ and $F_{\eta-(r)}$ determine the spectral-angular distribution at fixed intensity of the resonant differential cross section for channels $\mathrm{A}$ and $\mathrm{B}$, correspondingly:

$$
\begin{gathered}
F_{\eta \pm(r)}=\frac{\left(1-x_{\eta \pm(r)}\right) x_{\eta \pm(r)}}{g_{\eta \pm(r)}} P\left(u_{\eta \pm(r)}, \kappa_{\eta(r)}\right), \\
D_{\eta i}=\left(\frac{16 \pi \omega_{i}}{\alpha m}\right)^{2} \frac{1}{\left(1+\eta^{2}\right) \mathrm{K}^{2}(\eta)} .
\end{gathered}
$$

For the input parameters (34), we have:

TABLE I. The most probable values of the outgoing angles with corresponding particle energies and resonant differential cross section magnitudes [for $I \sim 10^{18} \mathrm{~W} \mathrm{~cm}^{-2}$ and parameters (34)].

\begin{tabular}{cccccc}
\hline \hline$r$ & Channel & $\delta_{\eta \pm(r)}$ & $E_{+}, \mathrm{GeV}$ & $E_{-}, \mathrm{GeV}$ & $R_{\eta \pm(r)}^{\max }, Z^{2} \alpha r_{e}^{2}$ \\
\hline 15 & $\mathrm{~A}$ & 0.436 & 25.003 & 24.997 & $3.814 \times 10^{11}$ \\
& $\mathrm{~B}$ & 0.436 & 24.997 & 25.003 & \\
16 & $\mathrm{~A}$ & 0.531 & 25.002 & 24.998 & $3.734 \times 10^{11}$ \\
& $\mathrm{~B}$ & 0.531 & 24.998 & 25.0024 & \\
17 & $\mathrm{~A}$ & 0.626 & 25.023 & 24.977 & $3.098 \times 10^{11}$ \\
& $\mathrm{~B}$ & 0.626 & 24.977 & 25.023 & \\
\hline \hline
\end{tabular}



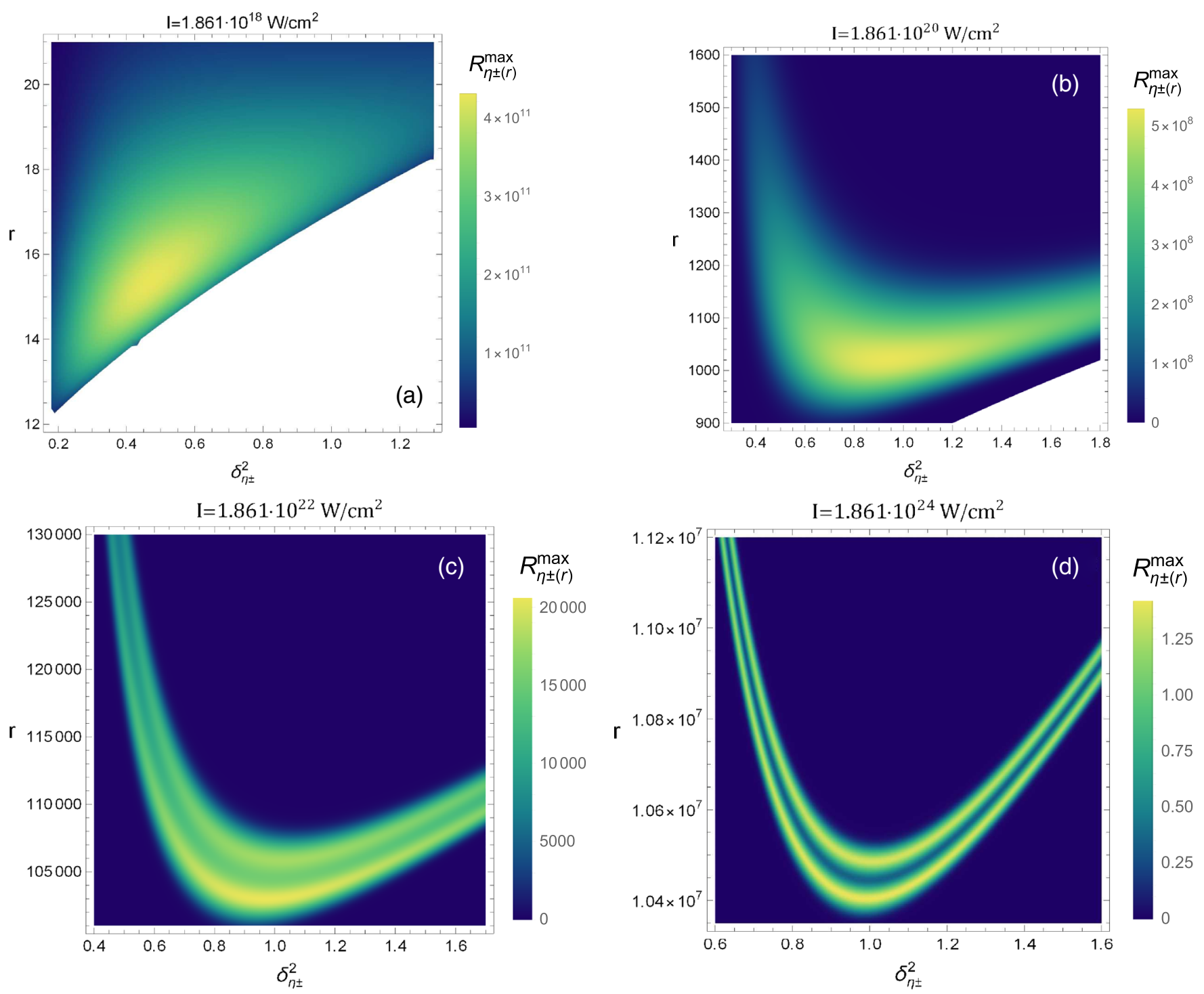

FIG. 5. The distribution of the maximum resonant differential cross section (73) with respect to the corresponding outgoing angle and amount of absorbed wave photons (in $Z^{2} \alpha r_{e}^{2}$ units) for different intensities and certain parameters (34).

$$
D_{\eta i} \approx 4.09 \times 10^{17} \frac{1}{\left(1+\eta^{2}\right) \mathrm{K}^{2}\left(r_{\eta}\right)}
$$

From Fig. 5(a)-Fig. 5(d) we can conclude, that only for particular values of the outgoing angles and number of absorbed wave photons resonant differential cross sections have significant magnitudes. In plane $\left(\delta_{ \pm}^{2}, r\right)$ these values form a region, which shape depends on the intensity. More detailed behavior of this region is presented in Fig. 4(a)-Fig. 4(d), where we can see that with increase in intensity, the number of maxima grows, and they become more distinguishable. Herewith, the global maximum exists for every value of intensity, and therefore it allows us to determine the most probable energies of produced particles and corresponding outgoing angles (see, for example, Table I). The maximum cross section magnitudes (in units

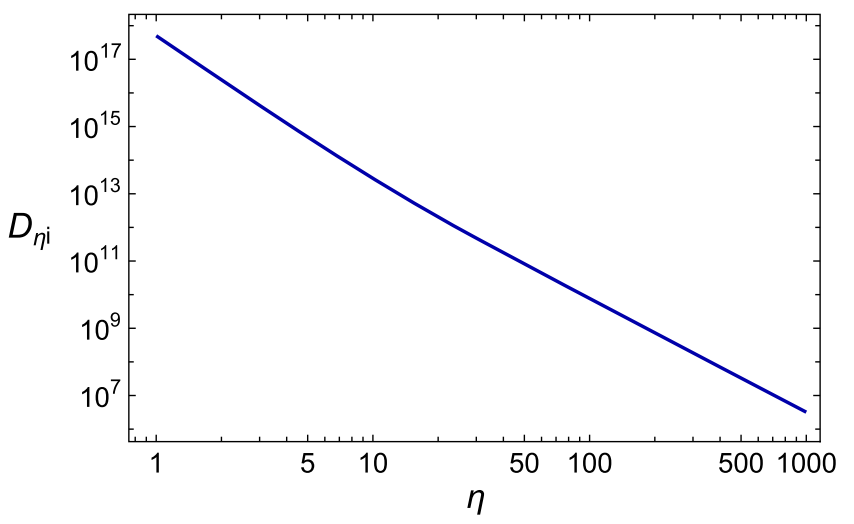

FIG. 6. The dependence of function $D_{\eta i}$ (75) on the classical relativistic parameter for the $\omega_{i}=50 \mathrm{GeV}$. 
$Z^{2} \alpha r_{e}^{2}$ ) are defined by the function $D_{\eta i}$ (see Fig. 6), that contains contributions from radiation width and small transferred momentum. The former increases with intensity growth and, therefore, the resonant differential cross section magnitude decreases. The values of the resonant differential cross sections themselves are of the order of $10^{11}, 10^{8}, 10^{4}$ and 1 (in $Z^{2} \alpha r_{e}^{2}$ units) for the intensities values $I \sim 10^{18}, 10^{20}, 10^{22}, 10^{24} \mathrm{~W} \mathrm{~cm}^{-2}$ respectively. It is noteworthy, that in the Fig. 4 and Fig. 5 we represented the corresponding expressions with high-energy solutions (29), (30) therein. The reason is that expressions with low-energy solutions are utterly suppressed in comparison to the highenergy ones and do not make any impact into the resonant differential cross section.

\section{CONCLUSION}

We have considered the resonant photoproduction of electron-positron pair on a nucleus within the strong electromagnetic field. The thorough examination of fourquasimomentum conservation laws along with resonant conditions allowed us to determinate one of the possible resonant kinematics. Due to the kinematic conditions, we have to demand ultrarelativistic energies of the produced particles and consequently sufficient energy of the initial gamma quantum. Besides, the propagation of the produced particles has to be enclosed within the narrow cone in the initial gamma quantum direction. We established, that there is minimal required amount of absorbed wave photons in resonance. This amount is completely determined by the experimental setup and increases proportional to the external field intensity. The resonant energies of produced particles were derived as the functions of outgoing angle and quantum parameter $\kappa_{\eta(r)}$. The corresponding dependencies possess two different values for the certain outgoing angle. In addition, there is maximum outgoing angle for every value of quantum parameter $\kappa_{\eta(r)}$.
We obtained the resonant differential cross section of laser-assisted $\mathrm{BH}$ process with simultaneous registration of the particle energies and the corresponding outgoing angle (positron for channel A and electron for channel B) for vast intensity range. Also, we verified that under the resonant conditions, obtained differential cross section factorizes into the product of differential probability of laserstimulated BW process and differential cross section of laser-assisted Mott scattering. Herewith, the most probable situation is intermediate particle scatters on nucleus without absorption or emission of wave photons. The obtained angular distribution has distinguishable maxima for each value of the absorbed wave photons. The number of maxima varies from one to four, depending on the number of absorbed photons and intensity. Moreover, there are global maxima of the resonant cross section with respect to the outgoing angle and number of absorbed wave photons for certain intensity value. This fact gives opportunity to determine the most probable energies of the particles and their outgoing angles, and thus to define a resonant process with high accuracy. Noteworthy, that with increase in the intensity, the resonant differential cross section decreases due to the growth of the resonant width. There are also kinematic regions, where resonant differential cross section is totally suppressed.

The above analysis was carried out with considering the model of a plane monochromatic electromagnetic wave, which allowed us to provide analytical investigation, but led us to the notorious divergence of the resonant cross section. We employed the phenomenological procedure to eliminate this divergence, whereas, we emphasized that there are other more elegant and, in a matter of fact, more rigorous ways to deal with this problem. The detailed analysis involving proper elaboration of the resonant infinity and influence of the interference between the different channels will be a subject of future research.
[1] A. Di Piazza, C. Müller, K. Z. Hatsagortsyan, and C. H. Keitel, Rev. Mod. Phys. 84, 1177 (2012).

[2] A. Gonoskov, T. Blackburn, M. Marklund, and S. Bulanov, arXiv:2107.02161.

[3] T. G. Blackburn, Rev. Mod. Plasma Phys. 4, 5 (2020).

[4] Y. I. Salamin, S. Hu, K. Z. Hatsagortsyan, and C. H. Keitel, Phys. Rep. 427, 41 (2006).

[5] F. Ehlotzky, K. Krajewska, and J. Z. Kamiński, Rep. Prog. Phys. 72, 046401 (2009).

[6] F. Cajiao Vélez, J. Kamiński, and K. Krajewska, Atoms 7, 34 (2019).

[7] V. I. Ritus, J. Sov. Laser Res. 6, 497 (1985).
[8] P. Zhang, S. S. Bulanov, D. Seipt, A. V. Arefiev, and A. G. R. Thomas, Phys. Plasmas 27, 050601 (2020).

[9] C. N. Danson, C. Haefner, J. Bromage, T. Butcher, J.-C. F. Chanteloup, E. A. Chowdhury, A. Galvanauskas, L. A. Gizzi, J. Hein, D. I. Hillier et al., High Power Laser Sci. Eng. 7, e54 (2019).

[10] J. W. Yoon, Y. G. Kim, I. W. Choi, J. H. Sung, H. W. Lee, S. K. Lee, and C. H. Nam, Optica 8, 630 (2021).

[11] I. C. E. Turcu, B. Shen, D. Neely, G. Sarri, K. A. Tanaka, P. McKenna, S.P. D. Mangles, T.-P. Yu, W. Luo, X.-L. Zhu et al., High Power Laser Sci. Eng. 7, e10 (2019). 
[12] K. A. Tanaka et al., Matter Radiat. Extremes 5, 024402 (2020).

[13] S. Weber et al., Matter Radiat. Extremes 2, 149 (2017).

[14] D. Papadopoulos, J. Zou, C. Le Blanc, G. Chériaux, P. Georges, F. Druon, G. Mennerat, P. Ramirez, L. Martin, A. Fréneaux et al., High Power Laser Sci. Eng. 4, e34 (2016).

[15] J. Bromage, S.-W. Bahk, I. A. Begishev, C. Dorrer, M. J. Guardalben, B. N. Hoffman, J. Oliver, R. G. Roides, E. M. Schiesser, M. J. Shoup III et al., High Power Laser Sci. Eng. 7, e4 (2019).

[16] J. Rossbach, J. R. Schneider, and W. Wurth, Phys. Rep. 808, 1 (2019).

[17] A. Gonoskov, A. Bashinov, S. Bastrakov, E. Efimenko, A. Ilderton, A. Kim, M. Marklund, I. Meyerov, A. Muraviev, and A. Sergeev, Phys. Rev. X 7, 041003 (2017).

[18] J. Magnusson, A. Gonoskov, M. Marklund, T. Z. Esirkepov, J. K. Koga, K. Kondo, M. Kando, S. V. Bulanov, G. Korn, and S. S. Bulanov, Phys. Rev. Lett. 122, 254801 (2019).

[19] X.-L. Zhu, T.-P. Yu, M. Chen, S.-M. Weng, and Z.-M. Sheng, New J. Phys. 20, 083013 (2018).

[20] V. Oleinik, J. Exp. Theor. Phys. 25, 697 (1967).

[21] V. Oleinik, J. Exp. Theor. Phys. 26, 1132 (1968).

[22] K. Krajewska, Laser Phys. 21, 1275 (2011).

[23] J. Bos, W. Brock, H. Mitter, and T. Schott, J. Phys. A 12, 715 (1979).

[24] S. P. Roshchupkin, A. A. Lebed', E. A. Padusenko, and A. I. Voroshilo, Resonant effects of quantum electrodynamics in the pulsed light field, in Quantum Optics and Laser Experiments, edited by S. Lyagushyn (Intech, Croatia, Rijeka, 2012), Chap. 6, pp. 107-156.

[25] S. P. Roshchupkin, Laser Phys. 6, 837 (1996).

[26] A. Hartin, arXiv:1701.02906.

[27] R. Ruffini, G. Vereshchagin, and S.-S. Xue, Phys. Rep. 487, 1 (2010).

[28] H. A. Bethe and W. Heitler, Proc. R. Soc. A 146, 83 (1934).

[29] G. Breit and J. A. Wheeler, Phys. Rev. 46, 1087 (1934).

[30] E. Lötstedt, U. D. Jentschura, and C. H. Keitel, New J. Phys. 11, 013054 (2009).

[31] A. Di Piazza, E. Lötstedt, A. I. Milstein, and C. H. Keitel, Phys. Rev. A 81, 062122 (2010).

[32] S. Augustin and C. Müller, Phys. Lett. B 737, 114 (2014).

[33] C. Müller, A. B. Voitkiv, and N. Grün, Phys. Rev. A 67, 063407 (2003).

[34] K. Krajewska and J. Z. Kamiński, Phys. Rev. A 82, 013420 (2010).

[35] B. Hafizi, D. F. Gordon, and D. Kaganovich, Phys. Rev. Lett. 122, 233201 (2019).
[36] N. R. Larin, V. V. Dubov, and S. P. Roshchupkin, Phys. Rev. A 100, 052502 (2019).

[37] N. R. Larin, V. V. Dubov, and S. P. Roshchupkin, Mod. Phys. Lett. A 35, 2040025 (2020).

[38] S. P. Roshchupkin, N. R. Larin, and V. V. Dubov, Laser Phys. 31, 045301 (2021).

[39] N. R. Larin, S. P. Roshchupkin, and V. V. Dubov, Universe 6, 141 (2020).

[40] F. V. Bunkin and M. V. Fedorov, Zh. Eksp. Teor. Fiz. 49, 1215 (1965) [Sov. Phys. JETP 22, 844 (1966)].

[41] A. A. Lebed', Laser Phys. Lett. 13, 045401 (2016).

[42] W. H. Furry, Phys. Rev. 81, 115 (1951).

[43] D. M. Volkov, Z. Phys. 94, 250 (1935).

[44] S. P. Roshchupkin, V. A. Tsybul'nik, and A. N. Chmirev, Laser Phys. 10, 1231 (2000).

[45] Y. B. Zel'Dovich, Sov. J. Exp. Theor. Phys. 24, 1006 (1967).

[46] S. P. Roshchupkin, V. V. Dubov, A. V. Dubov, D. V. Doroshenko, N. R. Larin, G. K. Sizykh, and V. D. Serov, in High Power Lasers and Applications, edited by J. Hein, T. J. Butcher, P. Bakule, C. L. Haefner, G. Korn, and L. O. Silva, Proceedings of the International Society for Optics and Photonics Vol. 11777 (SPIE, Bellingham, WA, 2021), pp. 40-55.

[47] J. Schwinger, Phys. Rev. 82, 664 (1951).

[48] V. Berestetskii, L. Pitaevskii, and E. Lifshitz, Quantum Electrodynamics (Elsevier Science, New York, 2012), Vol. 4.

[49] S. P. Roshchupkin and A. I. Voroshilo, Resonant and Coherent Effects of Quantum Electrodynamics in the Light Field (Naukova Dumka, Kiev, 2008).

[50] W. Becker and H. Mitter, J. Phys. Math. Gen. 9, 2171 (1976).

[51] A. Di Piazza and T. Pătuleanu, Phys. Rev. D 104, 076003 (2021).

[52] F. Mackenroth and A. Di Piazza, Phys. Rev. Lett. 110, 070402 (2013).

[53] D. Seipt and B. Kämpfer, Phys. Rev. D 85, 101701 (2012).

[54] A. Ilderton, Phys. Rev. Lett. 106, 020404 (2011).

[55] G. Breit and E. Wigner, Phys. Rev. 49, 519 (1936).

[56] A. V. Borisov, V. C. Zhukovskii, and P. A. Éminov, Sov. Phys. J. 23, 184 (1980).

[57] A. V. Borisov, V. C. Zhukovskii, A. K. Nasirov, and P. A. Éminov, Sov. Phys. J. 24, 107 (1981).

[58] A. Hartin, Int. J. Mod. Phys. A 33, 1830011 (2018).

[59] A. A. Mironov, S. Meuren, and A. M. Fedotov, Phys. Rev. D 102, 053005 (2020). 